\title{
POTENCIAL DE CONTAMINAÇÃO AMBIENTAL DE HERBICIDAS UTILIZADOS NA CULTURA DO MILHO
}

\author{
DÉCIO KARAM ${ }^{1}$, JÉSSICA ALINE ALVES SILVA ${ }^{2}$ e LUIZ LONARDONI FOLONI ${ }^{3}$ \\ ${ }^{1}$ Pesquisador - Embrapa Milho e Sorgo, C.P.151, CEP 35701-970, Sete Lagoas, MG. E-mail: \\ karam@cnpms.embrapa.br \\ ${ }^{2}$ Graduando em Engenharia Ambiental - Centro Universitário de Sete Lagoas, C.P.295, CEP \\ 35701-242, Sete Lagoas, MG. E-mail: jessicaalial@gmail.com \\ ${ }^{3}$ Eng $^{\circ}$ Agrônomo, Prof. Dr. Colaborador, Departamento de Água no Solo, Faculdade de \\ Engenharia Agrícola, Universidade Estadual de Campinas, Caixa Postal 6011, CEP 13083- \\ 970, Campinas, SP.E-mail: lfoloni@gmail.com
}

$\overline{\text { Revista Brasileira de Milho e Sorgo, v.8, n.3, p. 247-262, } 2009}$

RESUMO - Plantas espontâneas despontam como entraves à cultura do milho, e podem causar prejuízos de até $85 \%$ da produção. Dos métodos aplicáveis ao controle de tais espécies, sobressalta-se o químico, empregado em $65 \%$ da área cultivada com milho, no Brasil. É sabido que interações entre moléculas orgânicas e o ambiente podem ser prejudiciais aos gradientes da organização ecológica, requerendo, assim, estudos mais apurados com vistas a conhecer o comportamento e o destino ambiental de pesticidas. $\mathrm{O}$ modelo QIA (Quociente de Impacto Ambiental) mensura prejuízos advindos do uso de agroquímicos, quanto aos impactos sobre o trabalhador agrícola (aplicador/coletor), à biota e ao consumidor final. Este trabalho objetivou avaliar o potencial de contaminação de herbicidas utilizados na cultura do milho. Coletaram-se dados inerentes às características físico-químicas, ambientais e toxicológicas dos ativos, os quais foram parametrizados e empregados no cálculo do quociente de impacto ambiental. Os resultados permitiram observar que os ingredientes imazapic e imazapyr detêm maior potencial de contaminação ao ambiente, ao passo que isoxaflutole caracteriza-se pelo menor.

Palavras-chave: comportamento, destino ambiental, riscos, toxidez. 


\title{
POTENTIAL FOR ENVIRONMENTAL CONTAMINATION BY HERBICIDES USED IN MAIZE CULTURE
}

\begin{abstract}
Spontaneous plants are a severe problem in maize culture, and may cause a decrease in yield up to $85 \%$. Among the methods used to control such species the chemical is the most important, being used in 65 percent of the cultivated area in Brazil. It is known that interactions between organic molecules and environment can be harmful to the gradients of the ecological organization, thus requiring more refined studies in order to understand the behavior and the environmental fate of pesticides. The EIQ model (Environmental Impact Quotient) quantifies damages caused by the use of agrochemicals, regarding impacts on the agricultural worker (applicator/harvester), on the biota and on the final consumer. The present work objectified to evaluate the contamination potential of the herbicides used in maize. Data of physical-chemical, environmental and toxicological characteristics of the actives were collected, parameterized and used for the calculation of the environmental impact ratio. The results showed that imazapyr and imazapic presented the highest potential for environment contamination while isoxaflutole showed the lowest.
\end{abstract}

Key words: behavior, environmental fate, risks, toxicity.

A cultura do milho ocupa posição de destaque na economia brasileira e, em decorrência da área plantada e do volume colhido, estima-se, para a primeira safra de 2009/2010, uma produção de 33.000 mil toneladas (Conab, 2009). Todavia, sabe-se que as perdas de produtividade ocasionadas pela ocorrência de plantas daninhas nessa cultura podem atingir prejuízos da ordem de até $85 \%$ (Karam et al., 2008), diferindo em função da espécie, do grau de infestação, do tipo de solo e das condições climáticas, além do estádio de desenvolvimento da cultura em relação ao clima (Pires, 2005).
Dentre os métodos de controle de plantas daninhas no cultivo do milho, o químico ainda é o mais utilizado. Estima-se que o uso de herbicidas abranja aproximadamente $65 \%$ de toda a área cultivada com esse cereal, no Brasil (Karam et al., 2008). Atualmente, há, no Ministério da Agricultura Pecuária e Abastecimento (MAPA), registros de 35 ingredientes ativos para tal cultura, constituintes de formas isoladas ou misturas de 129 produtos formulados, pré ou pós-emergentes (BRASIL, 2009; AGÊNCIA, 2009). O glyphosate, à parte, compõe a lista por dispor de registro para uso no manejo de espécies daninhas na dessecação em pré-plantio do cultivo 
de milho. Cultivares de milho tolerantes a esse herbicida (geneticamente modificado) já foram liberadas para comercialização: entretanto, não há registro de uso desse herbicida para o controle de plantas daninhas nessa cultura.

É sabido que a sinergia entre moléculas orgânicas utilizadas para fins biocidas e ambiente pode ocasionar pernícies a todo o gradiente da organização biológica, tanto aos seus níveis superiores, tais como populações, comunidades e ecossistemas, quanto em escala individual, quando da detecção de alterações comportamentais, malformações, mudanças nas taxas de crescimento, reprodução, alimentação, alterações bioquímica e fisiológica, segundo as variáveis concentração e tempo de exposição (Arias et al., 2007). A compilação desses parâmetros compõe objeto de estudo que, pela toxicologia ambiental, atém-se ao destino final dos agentes tóxicos, seus metabólitos e demais produtos advindos de sua degradação no ambiente e cadeias tróficas, além de seus efeitos sobre organismos e populações (Costa et al., 2008), aliados à estatística e legislação (Arantes et al., 2008). Trabalhos diversos têm sido realizados com o intuito de predizer o comportamento ambiental de pesticidas e os riscos acerca de seu emprego (Gibson \& Koifman, 2008; Lira et al., 2009; Rodrigues et al., 2009; Metallo et al., 2008; Vivian et al., 2008; Cabrera et al., 2008). Tais indícios corroboram a distância entre informações no que tange à utilização, riscos e as consequências advindas do uso de herbicidas, recrutando o desenvolvimento de ferramentas que subsidiem a tomada de decisão por parte de trabalhadores rurais. Vários métodos têm sido desenvolvidos para o estudo e a previsão do comportamento e destino ambiental de agrotóxicos, particularmente de herbicidas, que vão desde o uso de índices e ferramentas constituídas de parâmetros preestabelecidos até modelos matemáticos. O uso de parâmetros, índices e modelos orienta tanto os trabalhos de campo como as análises laboratoriais (Spadotto, 2002), visando a aproximação das condições reais e boa reprodutibilidade dos modelos utilizados (Firmino et al., 2008). Não obstante, estudos acerca do potencial de contaminação de moléculas orgânicas, em suas mais distintas esferas, são ainda incipientes, uma vez que tais pesquisas exigem técnicas especializadas, além de apresentarem custo elevado, envolvendo o uso de elementos marcados e sofisticada instrumentação analítica (Pires et al., 2003).

O modelo QIA (Quociente de Impacto Ambiental), descrito por Kovach et al. (1992), mensura os impactos ambientais advindos do uso de agrotóxicos e pode ser definido como uma medida composta de vários parâmetros que contribuem para o impacto líquido e ambiental de um único ingrediente ativo (James, 2003). Os fatores utilizados na determinação do QIA para um ativo específico alicerçam-se em equações atinentes às seguintes categorias: riscos de contaminação do trabalhador-manuseador, riscos ecológicos de contaminação e riscos ao consumidor final, com o intuito de sumarizar os efeitos de tais 
compostos sobre os diversos compartimentos ambientais.

$\mathrm{Na}$ escolha do herbicida, o conhecimento do modo e do mecanismo de ação na planta, de sua degradação e persistência no solo, bem como dos níveis de toxicidade e possíveis riscos de contaminação da água, de alimentos e do próprio homem, são fundamentais para o monitoramento de seu impacto ambiental. Este trabalho teve como escopo a caracterização do potencial de contaminação dos principais herbicidas utilizados na cultura do milho, no Brasil, conforme seus efeitos de impacto ambiental.

\section{Material e Métodos}

\section{Consulta em base de dados}

A coleta de informações sobre os parâmetros constituintes do quociente de impacto ambiental deu-se, primeiramente, por meio de pesquisa em diferentes bases de dados e trabalhos científicos: Oliveira (2001); Inoue et al. (2003); Coutinho et al. (2005); Rodrigues \& Almeida (2005), dos quais se recolheram informações acerca dos 20 ingredientes ativos selecionados. Do AGROFIT (BRASIL, 2009), foram extraídas informações sobre os principais herbicidas registrados para a cultura do milho, bem como nomenclatura dos ativos com respectivos grupos químicos. Os ingredientes ativos selecionados para o cálculo de quociente de impacto ambiental foram: 2,4-D, alachlor, ametryn, amicarbazone, atrazine, bentazon, carfentrazone, foramsulfuron, glyphosate, imazapic, imazapyr, iodosulfuron, isoxaflutole, mesotrione, nicosulfuron, pendimethalin, simazine, s-metolachlor, tembotrione e trifluralin. Os dados requeridos no cálculo do QIA, para os ativos supracitados, foram obtidos em bancos de dados da PPDB (2009), AGENCE (2009), EXTOXNET (2009) e MSDS-Search (2008).

\section{Determinação do QIA}

O índice QIA foi determinado para cada herbicida por meio de vários componentes de risco ambiental. As variáveis empregadas foram: (DT) toxicidade dermal para mamíferos, dada na unidade $\mathrm{LD}_{50},(\mathrm{C})$ toxicidade crônica para mamíferos, unidade valor de NOEC, (SY) sistemicidade - sistêmica ou não, (F) toxicidade oral aguda para peixes $\mathrm{LC}_{50},(\mathrm{~L})$ potencial de lixiviação (GUS), (R) risco de deslocamento horizontal, Koc, (D) toxicidade oral aguda para pássaros, $\mathrm{LD}_{50},(\mathrm{~S})$ meia-vida no solo, $\mathrm{DT}_{50},(\mathrm{Z})$ toxicidade oral aguda para abelhas, $\mathrm{LD}_{50}$, (B) toxicidade para outros artrópodes benéficos, $\mathrm{LC}_{50}$, e $(\mathrm{P})$ meia-vida na planta, $\mathrm{DT}_{50}$.

Esses componentes foram divididos em três categorias: risco ao trabalhador-manuseador (RT), riscos sobre consumidores (RC) e riscos ecológicos (REc). A padronização dos dados consultados foi feita por meio de escala, de 
acordo com a gradação dos riscos, conforme o apresentado na Tabela 1, para as variáveis DT, C, SY, F, L, R, D, S, Z, B e P. Segundo
Kovach et al. (1992); a fórmula utilizada para o cálculo do quociente de impacto ambiental deve ser descrita como:

$$
Q I A=\left\{C\left[\left(D T^{*} 5\right)+\left(D T^{*} P\right)\right]+\left[\left(C^{*}((S+P) / 2) * S Y\right)+(L)\right]+\left[\left(F^{*} R\right)+\left(D^{*}((S+P) / 2) * 3\right)+\left(Z^{*} P * 3\right)+(B * P * 5)\right]\right\} 1 / 3 .
$$

TABELA 1. Parametrização das informações coletadas para uso no cálculo do quociente de impacto ambiental.

\section{MODO DE AÇÃO*}

não-sistêmica (1)

sistêmica (3)

LD50 DERMAL MAMÍFEROS (mg.Kg-1)

$>2000$ (1)

200 a $2000(3)$

0 a $200(5)$

NOEC

0 a 20 (1)

20 a $100(3)$

$>100(5)$

T ${ }_{1 / 2}$ PLANTA (SEMANAS)*

1 a 2 semanas (1)

2 a 4 semanas ( 3 )

$>4$ semanas (5)

$T_{1 / 2}$ SOLO*

$\mathrm{T} 1 / 2<30(1)$

$\mathrm{T} 1 / 2=30$ a $100(3)$

$\mathrm{T} 1 / 2>100(5)$

\section{LC50 AGUDA 96 h PEIXES(mg/L $\left.{ }^{-1}\right)$}

$>10$ ppm (1)

1 a 10 ppm (3)

$<10$ ppm (5)

LD50 AGUDA PÁSSAROS (mg.Kg-1 ${ }^{-1}$ *

$>1000$ ppm (1)

100 a $1000(3)$

1 a 100 ppm (5)

TOXICIDADE PARA ABELHAS*

baixo impacto (1)

médio impacto (3)

alto impacto (5)

TOXICIDADE PARA ORGANISMOS BENÉFICOS*

baixo impacto (1)

médio impacto (3)

impacto severo (5)

POTENCIAL DE LIXIVIAÇÃO (GUS)*

$<1,8$ (1)

1,8 a 2,8 (3)

$>2,8(5)$

\section{DESLOCAMENTO}

Koc $<300 \mathrm{KgL}-1, \mathrm{~T}_{1 / 2}>21$ (5)

Koc $>500 \mathrm{KgL}-1, \mathrm{~T} 1 / 2<14$ (1)

fora de enquadramento (3)

* Fonte: Kovach et al., (1992). 
Em que o risco à saúde do trabalhador (aplicador/coletor) agrícola foi definido como a soma dos riscos de exposição do aplicador (DT * 5) e coletor (DT * P) vezes possíveis efeitos crônicos $(\mathrm{C})$. O risco de exposição do aplicador foi representado pela toxicidade cutânea vezes cinco, que simboliza riscos associados à manipulação de pesticidas concentrados, enquanto o risco ao coletor (DT * P), foi representado pela toxicidade cutânea vezes a meia vida do ativo na superfície da planta. Este é multiplicado pelos efeitos crônicos potenciais (C). A equação que pormenoriza a categoria descrita acima é dada por:

$$
R T=C\left[\left(D T^{*} 5\right)+\left(D T^{*} P\right)\right]
$$

$\mathrm{O}$ fator de risco aos consumidores

foi definido pela soma do risco de exposição dos potenciais consumidores $(C *((S+P))$ 2) * $S Y$ ) e riscos de lixiviação dos ativos $(L)$, correlacionando as variáveis toxicidade crônica, meia-vida na planta, meia-vida no solo e sistemicidade do ingrediente:

$$
R C=C *((S+P) / 2) * S Y)+(L)
$$

A componente ecológica do modelo foi dada pela soma dos efeitos das substâncias químicas sobre biótopos aquático e terrestre. Ao produto dos riscos de intoxicação aguda oral para peixes e deslocamento superficial da substância $(\mathrm{F} * \mathrm{R})$, adicionaram-se riscos de intoxicação oral aguda para aves vezes a média da soma da meia-vida do ingrediente ativo no solo e nas plantas, multiplicado pelo coeficiente
3: $(D *((S+P) / 2) * 3)$. O risco para abelhas e artrópodes benéficos foi representado por $(Z *$ $P * 3)$ e $(B * P * 5)$. Esses relacionam os fatores toxicidade aguda oral para abelhas e artrópodes, meia-vida na planta e os coeficientes 3 e 5 . O impacto ambiental de pesticidas em sistemas aquáticos foi determinado pela multiplicação da toxicidade química do pesticida multiplicado pelo potencial de perda deste por escorrimento superficial. A semiequação representante dos riscos ecológicos foi descrita:

$$
R E c=(F * R)+(D *((S+P) / 2) * 3)+(Z * P * 3)+(B * P * 5)
$$

\section{Resultados e Discussão}

Dentre os ingredientes ativos objeto desse estudo, cinco são recomendados para aplicação em pré-emergência, nove em pós-emergência e cinco para ambos os casos e ainda o glyphosate, empregado como dessecante. Para Christoffoleti \& Mendonça (2001), os programas de manejo de plantas daninhas na cultura de milho que utilizam herbicidas pós-emergentes têm aumentado bastante nos últimos anos. Isso pode ser função do fato de herbicidas aplicados em pré-emergência terem sua eficiência comprometida em situação de umidade reduzida e alta quantidade de palha no solo (Karam et. al, 2008), ou, ainda, propiciado pela expansão de práticas agrícolas conservacionistas, como o plantio direto, que, segundo Gomes Jr. \& Christoffoleti (2008), ocupa atualmente 22 milhões de hectares de terras produtivas no Brasil. 
Imazapic e imazapyr (família das imidazolinonas) apresentaram maiores riscos de perturbação à saúde do trabalhador (Figura 1), 120 para ambos. Os produtos dessa classe, em mistura formulada, são recomendados em pós-emergência, para o controle de plantas daninhas de folhas largas, folhas estreitas e ciperáceas que infestam as lavouras de milho tolerante a esses produtos, sistema "clearfield" (Penckowski et. al, 2004). Imidazolinonas foram também apontadas por Kraemer et al. (2009) como compostos de alta persistência no solo.

Seu uso, no entanto, é de menor expressão, se comparado a de outros compostos inibidores de acetolactato sintase (ALS), como nicosulfuron (sulfonilureias), que alcançou índice de risco igual a 72, para a mesma categoria. Ferreira \& Carvalho (2005), em pesquisa sobre agrotóxicos utilizados na cidade de Sertanópolis, PR, relatam que apenas uma imidazolinona foi lembrada por ruralistas. Às também sulfonilureias foramsulfuron e iodosulfuron foram conferidos risco de perturbação à saúde do trabalhador iguais a 120 e 72, respectivamente. Compostos à base de sulfonilureias também deram ideia de possíveis poluidores de águas subsuperficiais (Dinelli et al., 1997), por apresentarem degradação lenta, em ensaios de dissipação e

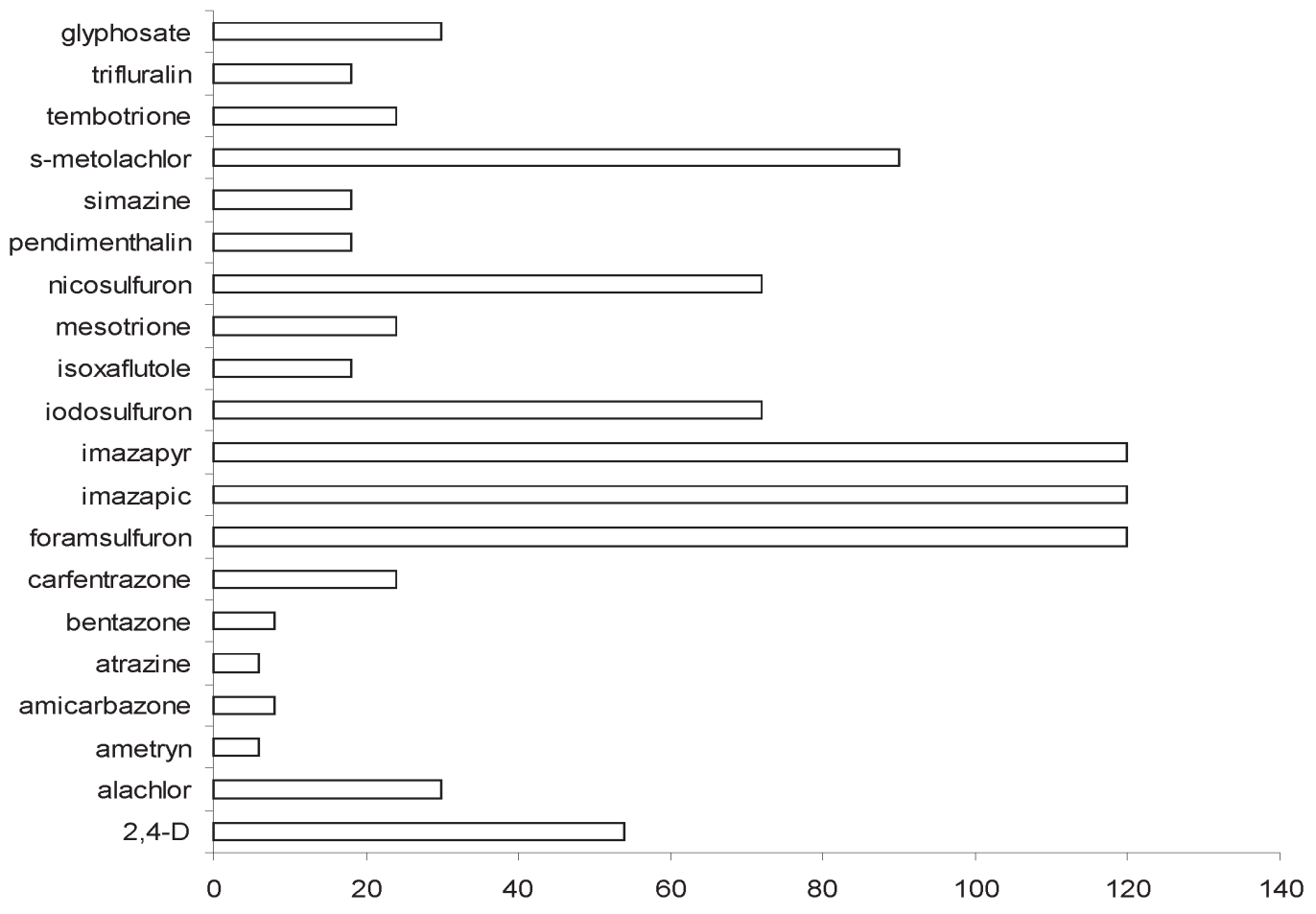

FIGURA 1. Classificação dos ingredientes ativos quanto aos ricos de contaminação ao trabalhador (aplicador/coletor). 
hidrólise in vitro. Nicosulfuron foi classificado como levemente nocivo à capacidade de parasitismo de fêmeas de Trichogramma pretiosum, em laboratório (Stefanello Júnior et al., 2008). O valor obtido na classe de risco ao trabalhador, para alachlor, foi 30 , à medida que para s-metolachlor foi de 90. As triazinas ametryn e atrazine representaram os mais baixos patamares de risco ao trabalhador, seis para ambas. Agentes herbicidas triazínicos foram descritos por Metallo et al. (2008) como detentores de baixo potencial de retenção por solos do bioma Mata Atlântica, assim como por Vivian et al. (2008), com o herbicida ametryn, que apresentou dessorção relevante, em ensaios com seis solos brasileiros. Resultado semelhante foi obtido por Cabrera et al. (2008), para 2,4-D, atrazine, glyphosate, pendimethalin e trifluralin, o que acentua o potencial de contaminação de águas superficiais e subterrâneas por estes. Segundo dados do SINITOX (2007), aproximadamente $70 \%$ dos casos de acidentes individuais pelo uso errôneo de agrotóxicos ocorreram naquele ano nas unidades federativas do Sul e do Sudeste. No mesmo ano, o SINDAG estimou que, por meio das vendas efetuadas, houve preponderância de herbicidas, dentre todos os agroquímicos, alcançando contingente de 2.685 milhões de reais, sendo incrementado em $45 \%$ no ano seguinte.

Quanto aos riscos ao consumidor (Figura 2), imazapic mostrou-se eminente, com risco definido igual a 65, seguido de imazapyr (35), foramsulfuron (33) e glyphosate (31). O não-atendimento ao período de carência requerido para cada molécula constitui risco de contaminação crônica da população consumidora de frutas e hortaliças com resquícios de agrotóxicos, uma vez que agricultores vêm pulverizando as frutas e verduras até o momento de serem comercializadas (Nina, 2002; Waichman et al., 2008). As moléculas amicarbazone, bentazon, carfentrazone e pendimethalin obtiveram nota 7, na mesma categoria.

Concernente aos riscos ecológicos, maiores valores foram encontrados para imazapyr (97), mesotrine (tricetonas: 97) e imazapic (87), respectivamente (Figura 3). Firmino et. al (2008) apontam o imazapyr como portador de alta mobilidade, em ensaios com três solos, tanto no sentido ascendente como no descendente, o que pode levar à contaminação de corpos d'água, bem como ocasionar ciclos de permanência do produto nas camadas mais superficiais e de subsuperfície, causando sérios danos ao ambiente (Pasqualetto et al., 2007). Kreutz et al. (2008) afirmam que níveis de toxicidade de xenobióticos, mensurada pela $\mathrm{LD}_{50}$, em alevinos de Rhamdia quelen, ficaram acima das concentrações efetivas aplicadas em lavouras. As moléculas isoxaflutole e s-metolachlor apresentaram os mais baixos valores de risco à ecologia: 20 .

Os valores totais obtidos da determinação do quociente de impacto ambiental estão detalhados na Tabela 2. O ingrediente ativo de 


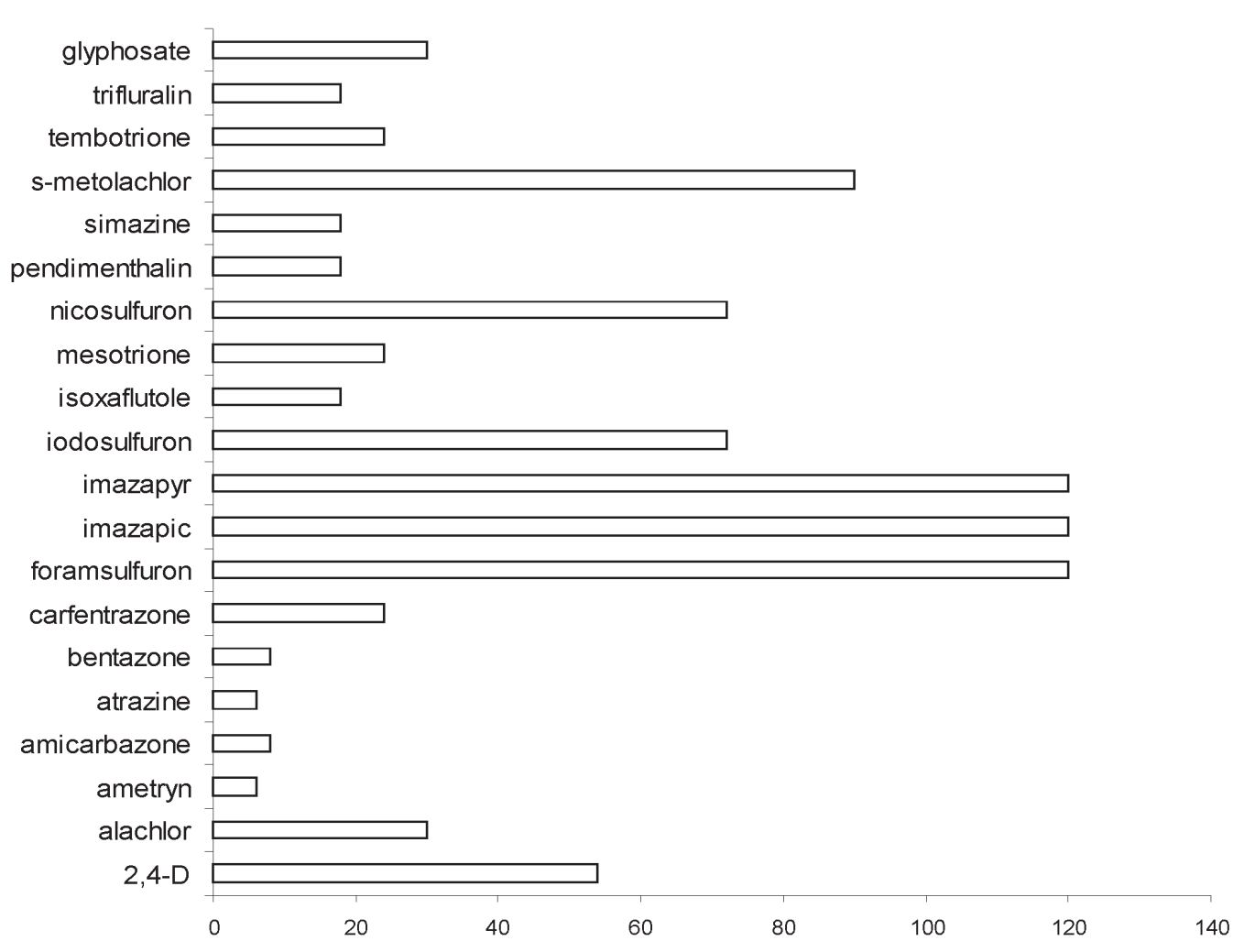

FIGURA 2. Classificação dos ingredientes ativos quanto aos riscos de contaminação ao consumidor final.

menor QIA foi isoxaflutole (14), pertencente à família isoxazol. Kowach et al. (1992) obtiveram QIA para a mesma substância de 22,7. Tal diferença pode estar associada a consultas de dados em fontes distintas. $\mathrm{Na}$ sequência, bentazon, ametryn e simazine resultaram nos menores valores do quociente, sendo 15,3, 16 e 16,7, respectivamente. Atrazine teve impacto calculado igual a 22. Dentre os ativos mais utilizados para o milho, as sulfonilureias e triazinas representaram, com nicosulfuron e atrazine, 30 e $65 \%$ de toda a área brasileira cultivada com essa cultura em que se utilizou herbicida na safra 2007/2008.
Embora o quociente de impacto observado para nicosulfuron seja cerca de três vezes maior que o encontrado para atrazine, devese salientar que a utilização dessa triazina ocorre em escala exponencial em todo o mundo, na forma isolada ou em associações, inclusive com nicosulfuron,. posto que atrazine é altamente persistente nos solos e tem mobilidade considerada de moderada a alta, em solos com pouco conteúdo de argila ou matéria orgânica (Cerdeira et. al, 2005). Tembotrione e mesotrione obtiveram índices iguais a 32 e 43,3, respectivamente (grupo químico das tricetonas), e mostraram QIA 


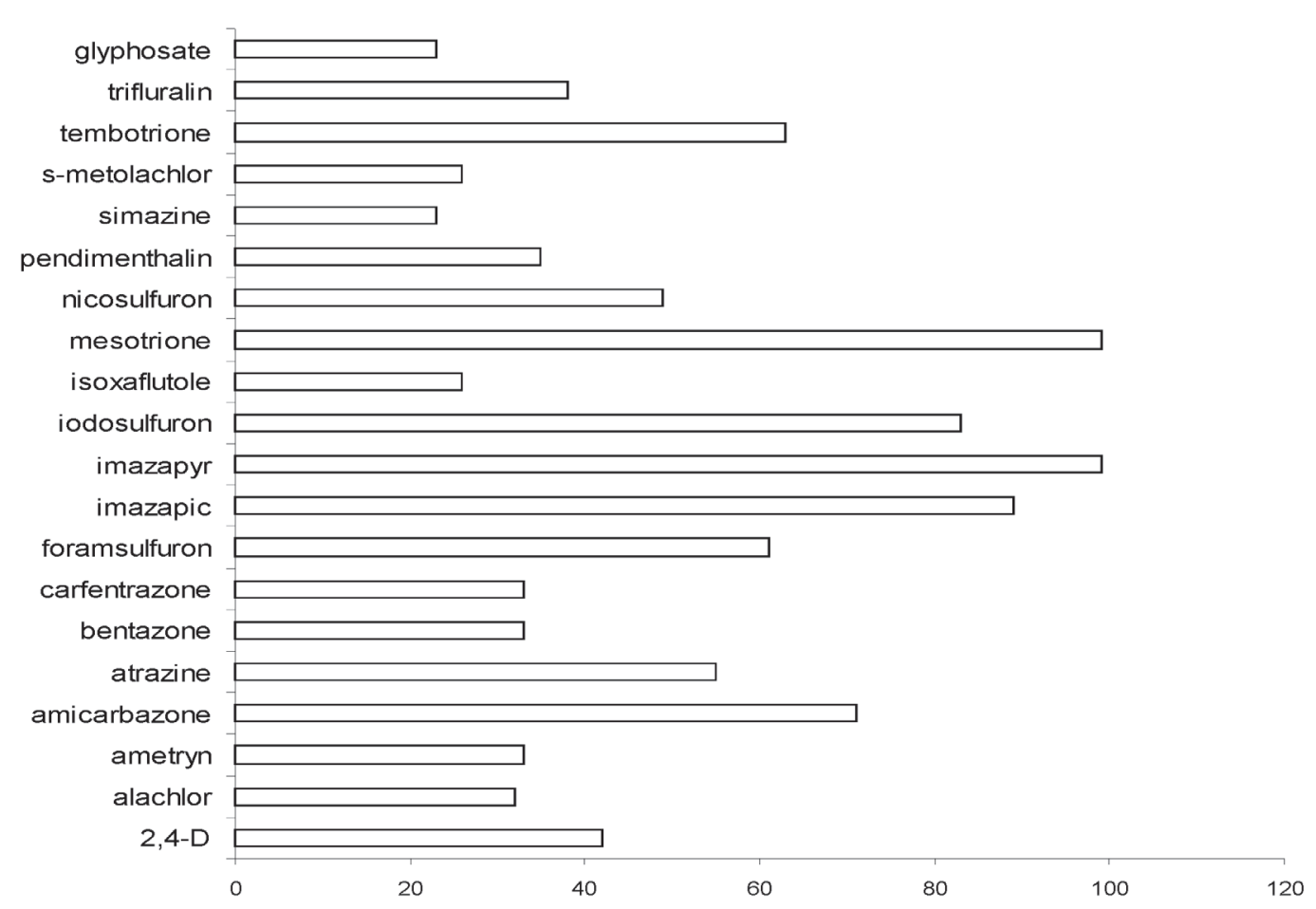

FIGURA 3. Classificação dos ingredientes ativos quanto aos riscos ecológicos.

mediano. Bonnet et al. (2008) encontraram menor potencial de toxicidade de moléculas tricetonas aos organismos Tetrahymena pyriformis e Vibrio fischeri, análogo a atrazine, por meio do teste de Microtox. O uso de tais moléculas tende a se expandir, visto que estas cingem grande número de espécies mono e dicotiledôneas infestantes de culturas diversas, como também pela baixa capacidade fitotóxica que denotam.

O glyphosate, constituinte da família química das glicinas substituídas, apresentou quociente de impacto de 28,7. Amarante Júnior et. al (2002) delegam a essa molécula pouca toxidez, mesmo havendo evidências de efeitos deletérios provenientes dela no ambiente, principalmente em detrimento da resistência adquirida por algumas espécies de ervas, após o uso prolongado do herbicida. Já Rissi et al. (2008), em estudo de cunho ecotoxicológico com o organismo-teste Daphinia similis e o herbicida glyphosate, sob duas concentrações, relataram a ausência de toxicidade aguda induzida naquele, em ambas as doses, ao passo que Albinati et al. (2009) detectaram hemorragia, necrose hepática e congestão renal em matrizes de Leporinus macrocephalus expostas a essa molécula.

Culturas tolerantes ao glyphosate representam a maior extensão dos cultivos transgênicos no mundo, reflexo do grande interesse comercial e das vantagens aos agricultores (James, 2003). Essa substância 
TABELA 2. Listagem de ingredientes ativos com respectivos grupos químicos e classificação segundo o Quociente de Impacto Ambiental.

\begin{tabular}{lll}
\hline \multicolumn{1}{c}{ Ingrediente ativo } & \multicolumn{1}{c}{ Grupo químico } & QIA \\
\hline $2,4-\mathrm{D}$ & Ácidos ariloxialcanoicos & 33,3 \\
Alachlor & Cloroacetanilidas & 25,3 \\
Ametryn & Triazinas & 16,0 \\
Amicarbazone & Triazolinonas & 27,3 \\
Atrazine & Triazinas & 22,0 \\
Bentazon & Benzotiadiazinonas & 15,3 \\
Carfentrazone & Triazolinonas & 23,3 \\
Foramsulfuron & Sulfonilureias & 71,3 \\
Glyphosate & Glicinas substituídas & 28,7 \\
Imazapic & Imidazolinonas & 90,7 \\
Imazapyr & Imidazolinonas & 84,0 \\
Iodosulfuron & Sulfonilureias & 56,3 \\
Isoxaflutole & Isoxazois & 14,0 \\
Mesotrione & Tricetonas & 43,3 \\
Nicosulfuron & Sulfonilureias & 48,0 \\
Pendimethalin & Dinitroanilina & 20,0 \\
Simazine & Triazinas & 16,7 \\
S-metolachlor & Acetanilidas & 40,0 \\
Tembotrione & Tricetonas & 32,0 \\
Trifluralin & Dinitroanilinas & 25,3 \\
\hline
\end{tabular}

tem comercialização crescente no Brasil, onde, segundo dados do IBAMA, ocorreu aumento sólido e significativo, no período de 2000 a 2005, com variações de até $80 \%$.

Todos os produtos vendáveis à base de imazapyr e imazapic registrados no MAPA, nas formas isolada ou constituinte de misturas, são classificados ambientalmente como muito perigosos e toxicologicamente como mediamente tóxicos, como também as formulações de isoxaflutole. Enquanto isso, atrazine abarca 6\% de produtos comerciais na classe dos altamente perigosos e $16 \%$ extremamente tóxicos. Detalhes acerca das classificações brasileiras ambiental e toxicológica podem ser visualizados na Figura 4. 

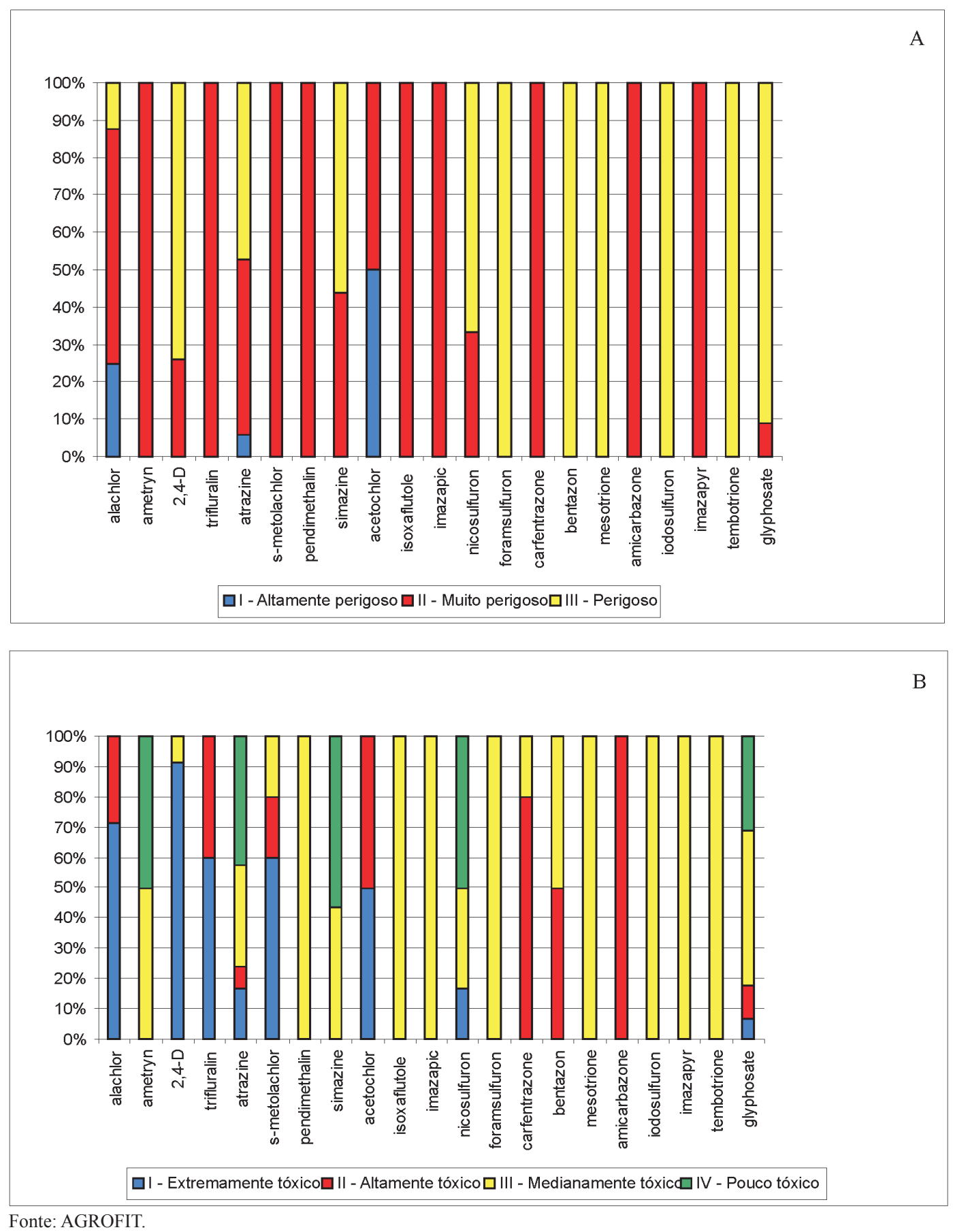

FIGURA 4. Classificação ambiental (A) e toxicológica (B) de herbicidas com registro no MAPA. 


\section{Conclusão}

Imazapic e imazapyr, indicados para a cultura do milho, possuem maior potencial de impacto ao meio ambiente, segundo seus possíveis riscos ambientais e ao homem, de acordo com o índice estimado através do quociente de impacto ambiental.

$\mathrm{O}$ uso de métodos que permitam caracterizar o comportamento de produtos fitossanitários no ambiente é de grande valia no que tange à tomada de decisão do agricultor sobre a escolha do produto químico a ser aplicado, permitindo que esse faça escolhas mais conscientes, tanto para a segurança na aplicação como para o meio ambiente.

\section{Literatura Citada}

AGENCE FRANCAISE DE SECURITÉ SANITAIRE DES ALIMENTS. AGRITOX: base de données sur les substances actives phytopharmaceutiques. Disponível em: http:// www.dive.afssa.fr/agritox/index.php. Acesso em: 29 abr. 2009.

AGÊNCIA NACIONAL DE VIGILÂNCIA SANITÁRIA. SIA: sistema de informações sobre agrotóxicos. Disponível em: http://www4. anvisa.gov.br/agrosia/asp/default.asp. Acesso em: 29 abr. 2009.

ALBINATI, A. C.; MOREIRA, E. L. T.; ALBINATI R. C. B.; CARVALHO, J. V.; A.D. DE LIRA, A. D. DE; SANTOS, G. B.; L.V.O.
VIDAL, L. V. O. Biomarcadores histológicos - toxicidade crônica pelo Roundup em piauçu (Leporinus macrocephalus). Arquivo Brasileiro de Medicina Veterinária e Zootecnia, Belo Horizonte, v. 8, p. 184-192, 2009.

AMARANTE JÚNIOR, O. P.; SANTOS, T. C. R. Glifosato: propriedades, toxicidade, usos e legislação. Química Nova, São Paulo SP, v. 25, n. 4, p. 589-593, 2002.

ARANTES, S. A. C. M.; LAVORENTE, A.; TORNISIELO, V. L. Efeito da calagem e do glifosato na atividade microbiana de diferentes classes de solos. Ecotoxicologia e Meio Ambiente, Curitiba, v. 17, p. 19-28, 2008.

ARIAS, A. R. L.; BUSS, D. F.; ALBURQUERQUE, C. DE; INÁCIO, A. F.; FREIRE, M. M.; EGLER, M.; MUGNAI, R.; BAPTISTA, D. F. Utilização de bioindicadores na avaliação de impacto e no monitoramento da contaminação de rios e córregos por agrotóxicos. Ciência e Saúde Coletiva. Rio de Janeiro, RJ, v.12, n. 1, p. 61-72, 2007.

BONNET, J. L.; BONNEMOY, F.; DUSSEL, M.; BOHATIER, J. Toxicity assessment of the herbicides sulcotrione and mesotrione toward two reference environmental microorganisms: Tetrahymena pyriformis and Vibrio fischeri. Archives of Environmental Contamination and Toxicology. New York, v. 55, p. 576-583, 2008.

BRASIL. Ministério da Agricultura, Pecuária e Abastecimento. Consulta de produtos formulados. Disponível em: http://extranet. 
agricultura.gov.br/agrofit_cons/principal_ agrofit_cons. Acesso em: 15 abr. 2009.

CABRERA, L. C.; COSTA, F. P.; PRIMEL, E. G. Estimativa de risco de contaminação das águas por pesticidas na região sul do estado do RS. Química Nova, São Paulo SP, v. 31, p. 1982-1986, 2008.

CERDEIRA, A. L. ; PESSOA, M. C. P. Y. ; SANTOS, N. A. G. ; LANCHOTE, V. L. Lixiviação de atrazina em solo em área de recarga do aqüífero Guarani. Revista Brasileira de Herbicidas, Passo Fundo, v. 2, p. 99-101, 2005 .

CHRISTOFFOLETI, P. J.; MENDONÇA, C. G. Controle de plantas daninhas na cultura de milho: enfoque atual. In: FANCELLI, A. L.; DOURADO-NETO, D. (Ed.). Milho: tecnologia e produtividade. Piracicaba: USP /ESALQ", 2001. p. 60-95.

CONAB. Acompanhamento da safra brasileira: grãos: segundo levantamento 2009 . Disponível em: http://www.conab.gov.br/ conabweb/download/safra/3graos_08.09.pdf. Acesso em: 17 nov. 2009.

COSTA, C. R. ; OLIVI, P.; BOTTA, C. M. R.; ESPINDOLA, E. L. G. A toxidade em ambientes aquáticos: discussão e métodos de avaliação. Química Nova, São Paulo SP, v. 31, n.7, p. 1820-1830, 2008.

COUTINHO, C. F. B.; TANIMOTO, S. T.; GALI, A.; GARBELLINI, G. S.; TAKAYAMA, M.; AMARAL, R. B.; MAZO, L. H.; AVACA, L. A.; MACHADO, S. A. S. Pesticidas: mecanismo de ação, degradação e toxidez. Pesticidas: revista de ecotoxicologia e meio ambiente, Curitiba, v.15, p. 65-72, 2005.
DINELLI, G.; VICARI, A.; BONETTI, A.; CATIZONE, P. Hydrolytic Dissipation of four sulfonylurea herbicides. Journal of Agricultural and Food Chemistry, Washington, v. 45, n. 5, p. 1940-1945, 1997.

EXTOXNET. EXtension Toxicology NETwork. Davis: University of California-Davis, 2009. Disponível em: http://extoxnet.orst.edu/. Acesso em: 29 abr. 2009.

FERREIRA, M. S.; CARVALHO, M. S. Agrotóxicos em Sertanópolis: tipos, uso e contaminação. Geografia, Londrina, v. 14, p. 99-113, 2005.

FIRMINO, L.E.; SANTOS, L. D.T.; FERREIRA, F. A.; FERREIRA, L.R.; TIBURCIO, R. A. S. Sorção do imazapyr em solos com diferentes texturas, Planta Daninha, Viçosa, MG, v. 26, n. 2, p. 395-402, 2008.

GIBSON, G.; KOIFMAN, S. Consumo de agrotóxicos e distribuição temporal da proporção de nascimentos masculinos no Estado do Paraná, Brasil. Revista Panamericana de Salud Publica, Washington, v. 24, n. 4, p. 240247, 2008.

GOMES JR., F. G.; CHRISTOFFOLETI, P. J. Biologia e manejo de plantas daninhas em áreas de plantio direto. Planta Daninha, Viçosa, MG, v. 26, n. 4, p. 789-798, 2008.

IBAMA. Relatórios de consumo de ingredientes ativos de agrotóxicos e afins no Brasil anos 1998 a 2001. Brasília, DF, 2003.

JAMES, C. Preview: Global Status of Commercialized Transgenic Crops: 2003. Ithaca: ISAAA, 2003. ( ISAAA Briefs, 30). 
KARAM, D.; GAMA, J. C. M. Radiografia dos herbicidas. Cultivar; grandes culturas, Pelotas, v. 63. p. 24-27, 2008.

KOVACH, J.; PETZOLDT, C.; TETTE, J. A method to measure the enviromental impact of pesticides. New York's Food and Life Sciences Bulletin, New York, v. 139, p. 1-8. 1992.

KRAEMER, A. F. et al. Comportamento ambiental dos herbicidas do grupo das imidazolinonas. Planta Daninha, Viçosa MG, v. 27, p. 629-639, 2009.

KREUTZ, L. C. et al. Acute toxicity test of agricultural pesticides on silver catfish (Rhamdia quelen) fingerlings. Ciência Rural, Santa Maria, v. 38, p. 1050-1055, 2008.

LIRA, S. V. et al. Intoxicações por pesticidas em crianças, adolescentes e jovens no município de Fortaleza (CE). Ciência, Cuidado e Saúde, Maringá, v. 8, n. 1, p. 48-75, 2009.

METALLO, M. B. et al. Sorption of $s$-triazines in brazilian rainforest soils. Ecotoxicologia e meio Ambiente, Curitiba, v. 18, p. 17-26, 2008 .

MSDS. Material Safety Data Factual Sheets Search. Disponível em: http://www. msdssearch.com/DBLinksN.htm. Acesso em: 12 jun. 2009.

NINA, N. C. S. Caracterização sócioeconômica-ambiental do uso de agrotóxicos em uma área de várzea do município de Manacapuru -Am (Amazônia Central). 2002. 123 f. Dissertação (Mestrado) - Universidade Federal do Amazonas, Manaus.
OLIVEIRA, M. F. Comportamento de herbicidas no ambiente. In: OLIVEIRA JR., R. S.; CONSTANTIN, J. (Ed.). Plantas daninhas e seu manejo. Guaíba: Agropecuária, 2001. p. 315-362.

PASQUALETTO, A.; ZITO, R. K. ; SILVA, A. A. ; RUIZ, H. A. Avaliação do lixiviado de Imidazolinonas por meio de bioensaio. Pesquisa Agropecuária Tropical, Goiânia, v. 29, n. 1, p. $1-5,2007$.

PENCKOWSKI, L. H.; PODOLAN, M. J.; LOPEZ-OVEJERO, R. F. Tolerância de milho tratado com inseticidas a herbicidas do grupo das imidazolinonas. Planta Daninha, Viçosa, MG, v. 22, p. 307-313, 2004.

PIRES, F. R.; SOUZA, C. M; SILVA, A. A; PROCÓPIO, S. O.; FERREIRA, L. R. Fitorremediação de solos contaminados com herbicidas Planta Daninha, Viçosa MG, v. 21, n. .2; p. 335-341. 2003.

PIRES, J. A. A. Plantio de milho com braquiária - Integração Lavoura-pecuária ILP. Viçosa: EMATER-MG, 2005. 5 p.

RISSI, T. A. et al. 2008. Teste ecotoxicológico antes e após a mineralização dos compostos glifosato, cihexatina e cipermetrina, utilizando o organismo-teste Daphnia similis. Centro Superior de Educação Tecnológica. Disponível em: http://cecemca.rc.unesp.br/ojs/index.php/ holos/article/viewFile/1528/1417. Acesso em: 12 jul. 2009.

RODRIGUES, B. N.; ALMEIDA, F. S. Guia de herbicidas. 5. ed. Londrina: IAPAR, 2005. 592 p. RODRIGUES, H. G. et al. Efeitos de pesticidas 
sobre a fragilidade osmótica de eritrócitos uma breve revisão. Biotemas, Florianópolis, v. 22, n. 1, p. 7-16, 2009.

SCORZA JUNIOR, R. P.; SILVA, J. P. Potencial de contaminação da água subterrânea por pesticidas na Bacia do Rio Dourados, MS. Pesticidas: Revista de Ecotoxicologia e Meio Ambiente, Curitiba, v. 17, p. 87-106, 2007.

SINDAG. Sindicato Nacional de Produtos para Defesa Agrícola. Disponível em: http://www. sindag.com.br/upload/ApresentacaoCTIAjansetembro08.ppt. Acesso em: 24 nov. 2009.

SINITOX - Sistema Nacional de Informações Toxicológicas. Casos registrados de intoxicação e/ou envenenamento 2004 Tabela 5. Disponível em: http://www.fiocruz. br/sinitox_novo/media/tab05_brasil_2004.pdf. Acesso em: 14 ago. 2009.

SPADOTTO, C. A. Comportamento e Destino Ambiental de Herbicidas. 2002. Comitê de Meio Ambiente, Sociedade Brasileira da Ciência das
Plantas Daninhas. Disponível em: http://www. cnpma.embrapa.br/herbicidas. Acesso em: 25 abr. 2009.

STEFANELLO JÚNIOR, G. J. et al. Seletividade de herbicidas registrados para a cultura do milho a adultos de Trichogramma pretiosum (Hymenoptera: Trichogrammatidae). Planta Daninha, Viçosa, MG, v. 26, p. 343-351, 2008.

PPDB - The Pesticide Properties Database - Agriculture \& Environment Research Unit (AERU). Disponível em: http://sitem.herts. ac.uk/aeru/footprint/en/index.htm. Acesso em: 4 abr. 2009.

VIVIAN, R, et al. Adsorção e dessorção de trifloxysulfuron-sodium e ametryn em solos brasileiros. Planta Daninha, Viçosa MG, v. 25, n. 1, p. 97-109, 2007.

WAICHMAN, A. V. Uma proposta de avaliação integrada de risco do uso de agrotóxicos no Estado do Amazonas. Acta Amazonica, Manaus, v. 38, p. 45-50, 2008. 